\title{
Adenine phosphoribosyltransferase (APRT) deficiency: identification of a novel nonsense mutation
}

\author{
Rea Valaperta ${ }^{1 * \dagger}$, Vittoria Rizzo ${ }^{2 \dagger}$, Fortunata Lombardi ${ }^{1}$, Chiara Verdelli ${ }^{1}$, Marco Piccoli ${ }^{3}$, Andrea Ghiroldi ${ }^{3}$, \\ Pasquale Creo ${ }^{3}$, Alessio Colombo ${ }^{4}$, Massimiliano Valisi ${ }^{4}$, Elisabetta Margiotta ${ }^{5}$, Rossella Panella ${ }^{6}$ and Elena Costa ${ }^{1,6}$
}

\begin{abstract}
Background: Adenine phosphoribosyltransferase deficiency (APRTD) is an under estimated genetic form of kidney stones and/or kidney failure, characterized by intratubular precipitation of 2,8-dihydroxyadenine crystals (2,8-DHA). Currently, five pathologic allelic variants have been identified as responsible of the complete inactivation of APRT protein.

Case presentation: In this study, we report a novel nonsense mutation of the APRT gene from a 47-year old Italian patient. The mutation, localized in the exon 5, leads to the replacement of a cytosine with a thymine (g.2098C > T), introducing a stop codon at amino acid position 147 (p.Gln147X).

This early termination was deleterious for the enzyme structural and functional integrity, as demonstrated by the structure analysis and the activity assay of the mutant APRT protein.
\end{abstract}

Conclusion: These data revealed that the p.G $\ln 147 X$ mutation in APRT gene might be a new cause of APRT disease.

Keywords: APRT deficiency, Renal failure, Crystalline nephropathy

\section{Background}

Adenine phosphoribosyltransferase deficiency (APRTD) is a rare autosomal recessive metabolic disorder due to a mutation of the APRT gene [1]. APRT is a purinemetabolism enzyme that catalyzes the formation of $5^{\prime}$ adenosine monophosphate (5'-AMP) and pyrophosphate (PP) from adenine and 5-phosphoribosyl-1-pyrophosphate $[2,3]$. In patients with complete APRT deficiency, adenine is oxidized by xanthine oxidase $(\mathrm{XO})$ to the highly insoluble and nephrotoxic derivative 2,8-dihydroxyadenine (2,8-DHA) [4], leading to urolithiasis and renal failure caused by intratubular crystalline precipitation [5,6]. The APRT gene, located on chromosome 16q24 [7], is approximately $2.6 \mathrm{~kb}$ long, contains five exons and four introns, and encodes a protein of 180 amino acid residues [8]. The human enzyme, present in a variety of cell types including erythrocyte [9], is a homodimer composed of

\footnotetext{
* Correspondence: rea.valaperta@grupposandonato.it

${ }^{\dagger}$ Equal contributors

${ }^{1}$ Research Laboratories - Molecular Biology, IRCCS Policlinico San Donato, Piazza E. Malan 2, 20097, San Donato Milanese, Milan, Italy

Full list of author information is available at the end of the article
}

two identical subunit species with a molecular weight of about 19.481 Da [10]. Currently, there are two isoforms produced by alternative splicing: the isoform 1 (P07741-1) and the isoform 2 (P07741-2); the isoform 1 has been considered as the 'canonical' one.

In the pathologic allelic variants, more than 40 mutations have been identified in the coding region of APRT gene in over 300 affected individuals from more than 25 countries, including at least 200 individuals from Japan. Approximately $10 \%$ of mutant alleles in affected white individuals and $5 \%$ in affected Japanese haven't been yet identified. APRT gene alterations include missense, frameshift, and nonsense mutations and small deletions/insertions ranging in size from 1 to 8 base pairs. The estimated heterozygosity in different populations ranges from 0.4 to $1.2 \%$ [11], suggesting that the prevalence of a homozygous state is at least 1:50,000 to $1: 100,000$.

Mutant alleles responsible for the disease have been classified as APRT*Q0 for type I and APRT*J for type II APRTD. Type I APRT deficiency (complete deficiency 
in vivo or in vitro) has been found in patients from many different countries [12-15]. Type II deficiency (complete enzyme deficiency in vivo but partial deficiency in cell extracts) has been found mainly in Japan [16-18]. However, this distinction is only of historical interest, because APRT enzyme activity in intact cells has been shown to be approximately $1 \%$ in both types [19].

The most common mutations in affected European individuals are: (i) $\mathrm{T}$ insertion at the intron 4 splice donor site (IVS4 + 2insT) which leads to the deletion of exon 4 from the mRNA because of aberrant splicing. This mutation has been found in individuals from many European countries as well as in an affected individual from the US, (ii) A-to-T transversion in exon 3 (g.194A > T, p.Asp65Val), described in affected individuals from Iceland, Britain, and Spain. The three most common mutations in affected Japanese individuals, in order of decreasing frequency, are: (i) T-to- $\mathrm{C}$ missense mutation in exon 5 (g.442 $\mathrm{T}>\mathrm{C}$ ), (ii) G-to-A nonsense mutation in exon 3 (g.329G > A) and (iii) a four-base pair (CCGA) duplication in exon 3 that leads to a frameshift after codon $186[20,21]$.

In the present study, we report the identification of a new nonsense mutation (g.2098C $>\mathrm{T}$ ) in exon 5 (p.Gln147X) of the APRT gene from an Italian patient affected by APRT deficiency.

\section{Case presentation}

\section{Clinical history of the patient}

The patient, born in 1964, was diagnosed as affected by obstructive chronic kidney disease (CKD) with crystalluria at the age of 28 . The serum creatinine was $4 \mathrm{mg} / \mathrm{dl}$. The composition of the crystals was not investigated. Treatment with allopurinol and bicarbonate resulted in modest and transient improvement of renal function.

In 2005, the patient started hemodialysis due to end stage renal failure. In April 2010, at the age of 46, he received a kidney transplant from a deceased donor. However, the disease rapidly recurred in the transplanted organ on the $9^{\text {th }}$ day after the transplant and the concentrations of creatinine and urea were $7.7 \mathrm{mg} / \mathrm{dl}$ and $204 \mathrm{mg} / \mathrm{dl}$, respectively. Two weeks after kidney transplant, a renal biopsy was performed and showed chronic tubulointerstitial nephropathy. Urinary sediment showed precipitations typical of 2,8-DHA crystals. After the diagnosis of APRT deficiency the allopurinol dose was increased to $300 \mathrm{mg}$ twice a day. The patient was dismissed on May 2010 with a $2 \mathrm{mg} / \mathrm{dl}$ concentration of creatinine. In October 2010, he was again hospitalized for a bacterial lung infection. The patient's general conditions worsened because of the onset of a multiorgan dysfunction and septic shock. The patient died in 2011, 10 months after the transplantation.

\section{Diagnosis of APRT deficiency}

The diagnosis of APRT deficiency disease in our patient was confirmed by: (i) the absence of APRT enzyme activity in erythrocytes, (ii) the characterization of 2,8DHA crystals in the urinary sediment and in the renal biopsy, (iii) the measurement of levels of adenine in a 24-hour urine specimen, (iv) the molecular analysis of the APRT gene.

High performance liquid chromatography (HPLC) analysis was used to measure APRT enzymatic activity in erythrocyte lysates and the levels of adenine were measured in 24-hour urine with UV detection [22].

The APRT activity was determined by calculating the AMP produced by hemolysates during the incubation with substrates over the basal hemolysate value, measured in a PRPP starved reaction.

The APRT activity in the patient was about $1 \%$ : residual APRT activity was $0.8 \mu \mathrm{mol} / \mathrm{min} / \mathrm{mg}$ of hemoglobin while in control individuals the mean value was $36.1 \pm 3.2 \mu \mathrm{mol} /$ $\mathrm{min} / \mathrm{mg}$. The concentration of adenine in the patient's urine was $15.68 \mathrm{mmol}$ per mol of creatinine while in the control range it is $0.18-0.20 \mathrm{mmol}$ per mol of creatinine.

Light microscopy analysis a kidney section from a biopsy taken two weeks after transplantation, presented irregular aggregates in most of the tubules in the renal cortex. The analysis by polarized microscopy showed precipitation of 2,8-DHA crystals within tubular lumen and interstitium (Figure 1A). At the same time, all urine samples were positive for small and medium sized 2,8-DHA crystals. Microscopic examination revealed multiple spherical and reddish-brown particles with a dark outline and central spicules, that look like "Maltese crosses" under polarized light. The 2,8-DHA crystals had atypical appearances with unusual birefringence features (Figure $1 \mathrm{~B}$ and $\mathrm{C}$ ).

\section{Molecular analysis}

Direct DNA sequencing, by Sanger method, of the five exons and their flanking regions showed that our patient was homozygous for a polymorphism in flanking region 2 named $r s 8191483$. He was also carrying a homozygous nonsense mutation in exon 5 in position g.2098C > T that introduced a stop codon at amino acid position 147 (p.Gln147X). The sequence analysis of the APRT gene from a healthy donor was used as control (Figure 2A). The homozygous nature of this site was confirmed by the presence of only one peak in the chromatogram on both strands of genomic DNA (Figure 2B). The $\mathrm{C}>\mathrm{T}$ replacement at this site leads to the formation of a truncated protein of 147 amino acids, compared to the normal 180 amino acid protein (Figure 2C). The Gln147X mutation has not been reported in previous studies, suggesting that this is a new nonsense mutation. Fifty healthy donor were used as controls. The p.Gln147X mutation was not found in any of them. The nomenclature used to 


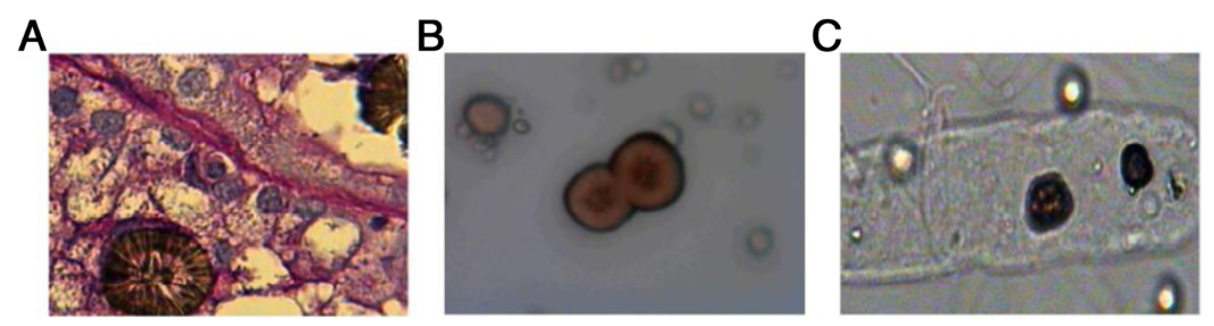

Figure 1 Characterization of 2,8-DHA crystals. (A) renal biopsy. Haematoxylin and myosin staining of a frozen section reveals interstitial inflammation surrounding multiple intra-tubular round brownish crystal that are birefringent under polarized light. (B, C) Urinary miscroscopy. Urinary sediment showing spherical brownish crystal that have a birefringent aqnd pseudo-Maltese cross appearance under polarized light. Magnifications: $\times 100$ in $\mathbf{A}, \times 40$ in $\mathbf{B}$ and $\mathbf{C}$.

describe the sequence variants was as recommended by Den Dunnen and Antonarakis [23] and by the Human Genome Variation Society (HGVS).

Unfortunately, the pedigree has not been investigated in our study since the family members refused genetic investigation.

\section{Protein analysis}

Erythrocytes isolation from peripheral blood of a control individual and from our patient was performed by Ficoll/ Paque density separation protocol. Purity was confirmed by flow cytometry with a mouse anti-human-CD45 PE (Figure 3A) and anti-CD235a PerCP-eFluor710 (Figure 3B) monoclonal antibodies. SDS-PAGE protein analysis revealed the lack of the $\mathrm{C}$-terminal domain in the patient sample compared to control. The N-terminal domain, used as internal control, was present in both samples (Figure 3C). Western blot analysis was performed using rabbit polyclonal APRT antibody (C-term) (Abgent, Cat AP2893b) and rabbit polyclonal APRT antibody (N-term) (Abgent, Cat AP2893a).

To analyze the structural impact of the p.Gln147X nonsense mutation on APRT, the 3-D structure of the protein was modeled based on the solved protein structure (PDB ID: 1ORE) using Swiss-PdbViewer software. We reported the loss of the C-terminal of the APRT protein which includes a portion of the S8 strand, the S9 strand and the H6 $\alpha$-helix, due to the new identified nonsense mutation (Figure 4A). The lack of this portion of the protein led to the removal of Leu159 residue, present in the protein active site (Figure 4B).

To evaluate the hydropathicity of both the wild type and the mutated protein, the Grand Average of Hydropathicity (GRAVY) was calculated with ProtParam Tool [24]. Loss of the C-terminal domain also caused an alteration in the protein hydrophobicity: the GRAVY of the wild type protein is 0.104 , whereas the GRAVY of the mutated protein is 0.058 , indicating that the mutated form of the protein is more hydrophilic than the wild type one.

\section{Conclusion}

APRT deficiency is a disease caused by mutations in the APRT gene. There are more than 40 types of mutations in APRT gene, but currently only five allelic variants are responsible for the complete inactivation of the APRT protein both in vitro and in vivo.

This disease is characterized by the presence of 2,8 DHA crystals in urine. These crystals are radiolucent and are often considered as uric acid stones or, in renal biopsies, as oxalate crystals, causing an erroneous diagnosis. Ultraviolet or infrared spectrophotometry is required for their correct identification.

Accurate diagnosis of APRT deficiency is crucial because early treatment with allopurinol or low-purine diet effectively prevents the stone formation and may improve patients' renal function. Nowadays, several diagnostic tools are available for the identification of the APRT deficiency such as 2,8-DHA crystal microscopic detection, APRT enzyme activity assays and molecular analysis of the APRT gene. In the present study, we analyzed an Italian patient with a clinical history of recurrent nephrolithiasis and chronic renal failure due to obstructive nephropathy and recurrent urinary tract infections. The patient received a renal transplant in 2010 but, unfortunately, he died 10 months after the surgical procedure. The definitive diagnosis of APRT deficiency was made by microscopic detection of 2,8-DHA crystals on the biopsy of the failing transplanted kidney and by documenting decreased APRT activity in red blood cells. Genetic analysis revealed a combination of a previously described homozygous state for $r s 8191483$ SNP and a novel nonsense mutation p.Gln147X. The identification of two allelic variants in homozygous state suggest the consanguinity in this family. The carriers are asymptomatic and they are usually identified during family screening.

The normal human APRT is a protein of 180 amino acids, composed of $9 \beta$-strands and $6 \alpha$-helices, which can be divided into the "core" (residues 33-169), the "hood" (residues 5-34), and the "flexible loop" (residues 95-113) domains. On the basis of the APRT structure, 


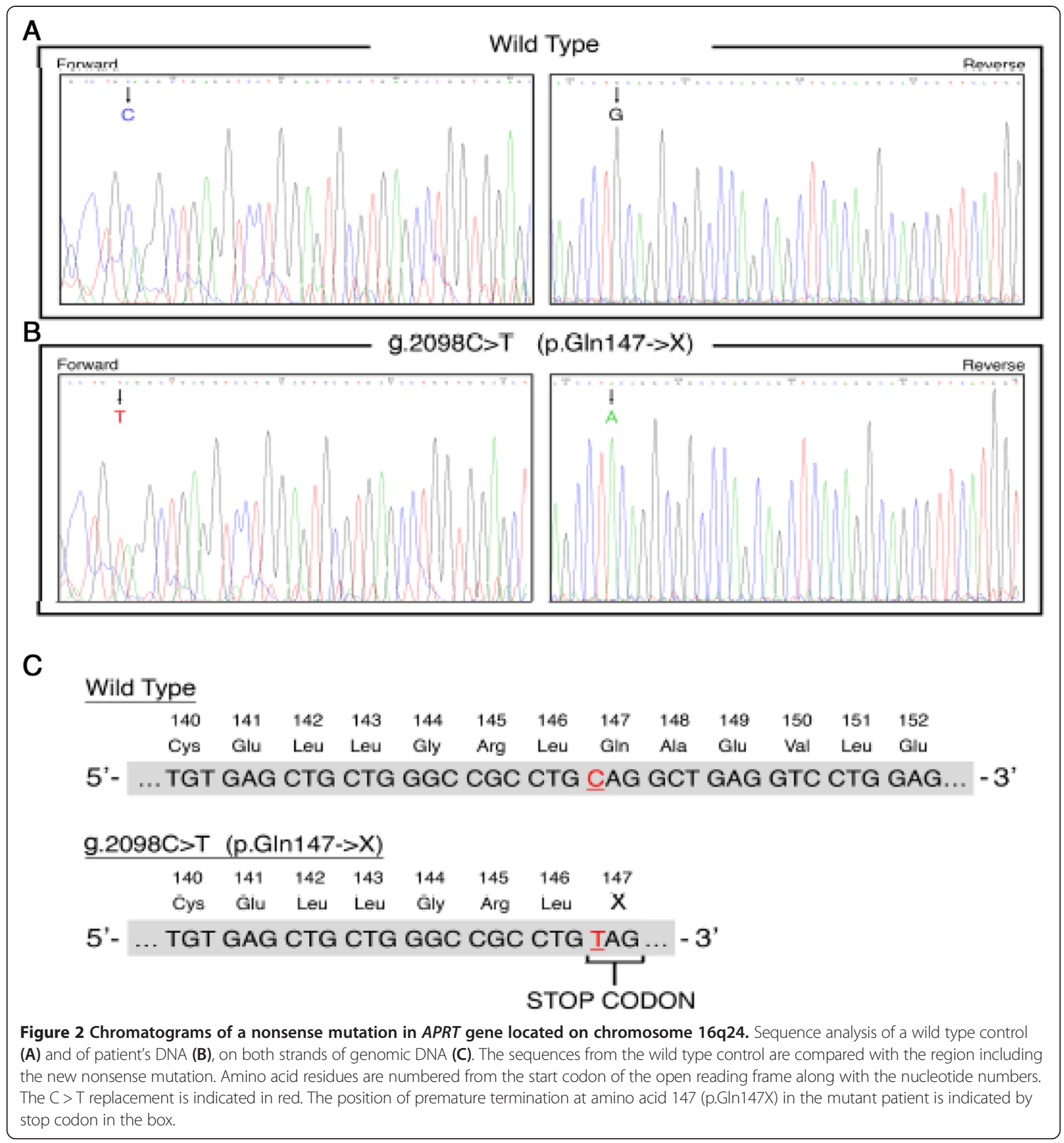

which has been widely described [21], it is evident that Ala131 and Leu159 are essential residues for the specific recognition of adenine among different purines through hydrophobic interactions. Moreover, the importance of the Leu159 residue is also confirmed by the presence in the same position of a lysine residue in the PRTases that binds hypoxanthine, xanthine, or guanine. This difference between residues located at the amino acid 159 position explains the specificity of type I PRTases for their respective purines [25].

The novel nonsense mutation reported in this study caused the introduction of a stop codon leading to the loss of 33 amino acids, corresponding to the C-terminal domain of the APRT protein. This portion represents the "core" of the APRT protein. In particular, the fundamental Leu159 residue, responsible for the correct activity 

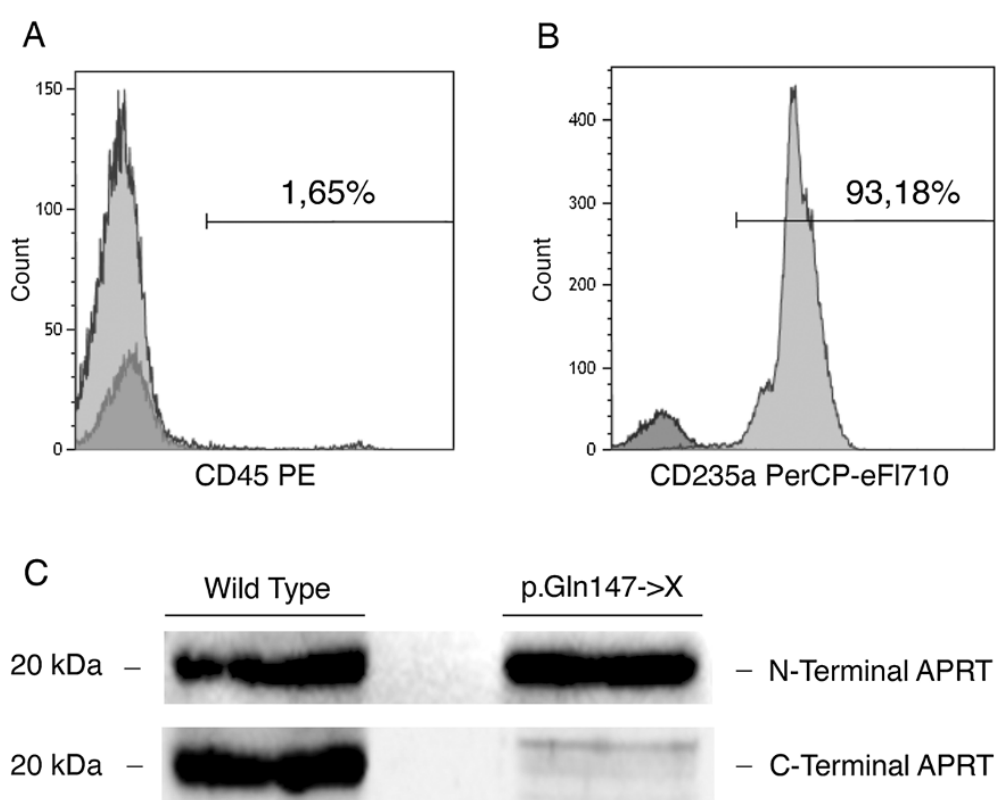

Figure 3 Characterization of APRT protein in erythrocyte lysate. Erythrocyte isolation from peripheral blood of a control and of patient by Ficoll/Paque density separation protocol. Purity was confirmed in flow cytometry, staining target cells with mouse anti-human anti-CD45 PE (leukocyte marker) (A) and anti-CD235a PerCP-eFluor710 (erythrocyte marker) monoclonal antibodies (B). (C) Western blot analysis performed by C-terminal and N-terminal APRT antibodies in a wild type control and in our patient.

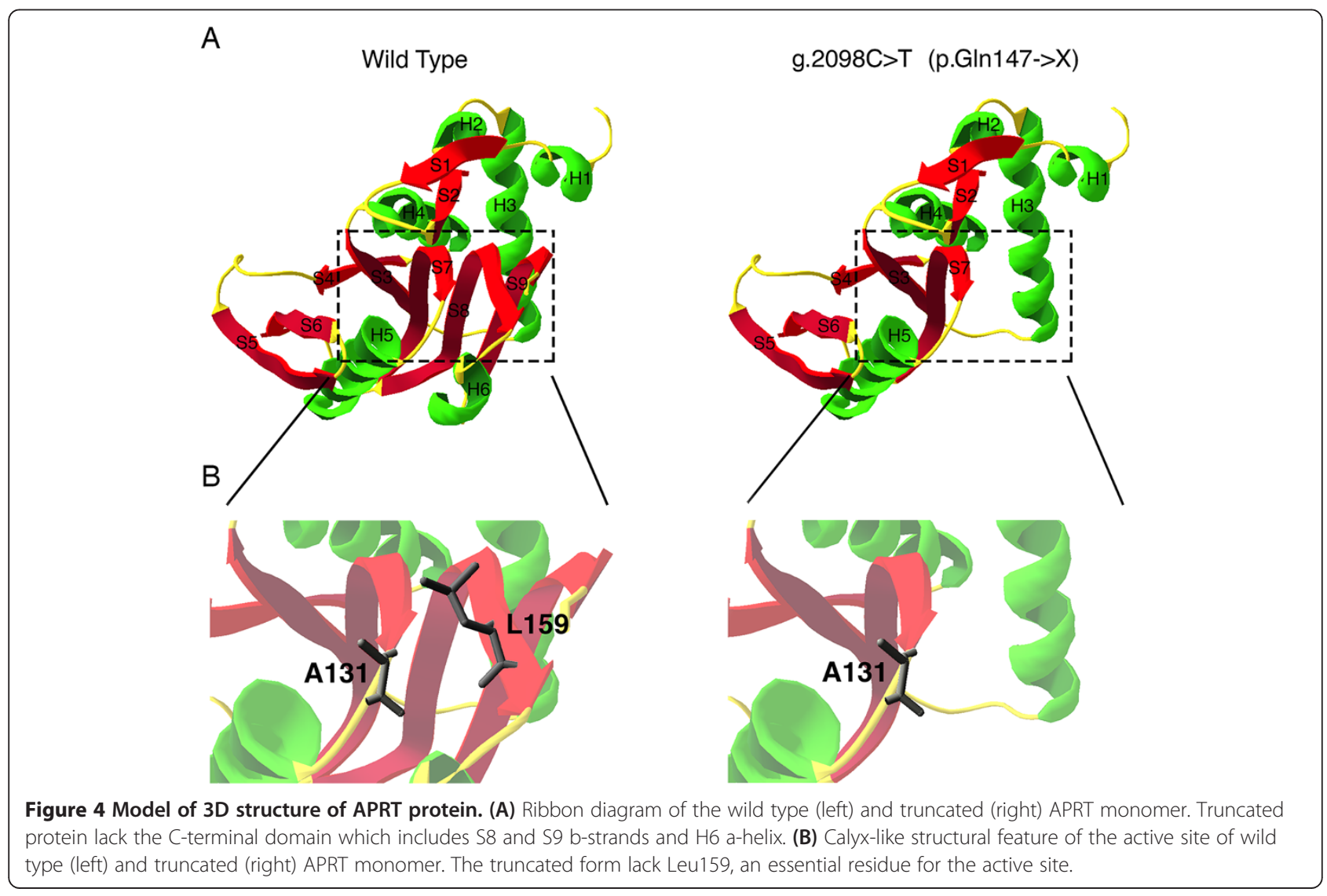


of the APRT enzyme, is located in the S8 strand. Therefore, the complete inactivation of the APRT protein reported in our patient is due to the impaired binding of the specific substrate to the active site, since the g.2098C > T mutation deeply affects the enzyme structural integrity.

In conclusion, the novel nonsense mutation p.Gln147X might be the main cause for the therapeutic failure observed in our patient.

Moreover, the evidence of this novel "loss-of-function" mutation might be extremely useful as a new genetic diagnostic marker for the early identification of the APRT deficiency.

More than 30 years have passed since the recognition of the first mutation in the APRT gene but, although much has been done during this period, the identification and characterization of new mutations might be an important step forward in the clinical practice for improving the detection of this still underdiagnosed disease.

\section{Consent}

Written informed consent was obtained from the patient for publication of this case report and any accompanying images. A copy of the written consent is available for review by the Editor of this journal.

\section{Abbreviations \\ APRTD: Adenine phosphoribosyltransferase deficiency; APRT: Adenine phosphoribosyltransferase; 2,8-DHA: 2,8-dihydroxyadenine crystals; GRAV Grand average of hydropathicity; HGVS: Human Genome Variation Society.}

\section{Competing interests}

No conflicts of interest, financial or otherwise, are declared by the author(s).

\section{Authors' contributions}

$R V, V R$ and FL conception and design of research; VR, FL, CV, MP, AG, PC, EM and RP performed experiments; $A C$ and MV performed sequencing; RV, VR, FL, EC analyzed data. All authors have contributed significantly to the work and have read and approved the manuscript.

\section{Acknowledgments}

This study was supported by IRCCS Policlinico San Donato and by IRCCS Policlinico San Matteo.

We thank Dr. Claudia Alpini and Dr. Sabrina Peressini for microscopy technical support.

\section{Author details}

${ }^{1}$ Research Laboratories - Molecular Biology, IRCCS Policlinico San Donato, Piazza E. Malan 2, 20097, San Donato Milanese, Milan, Italy. ²Department Molecular Medicine, Clinical Biochemistry Unit, Foundation IRCCS Policlinico San Matteo, University of Pavia, Pavia, Italy. ${ }^{3}$ Laboratory of Stem Cells for Tissue Engineering, IRCCS Policlinico San Donato, Milan, Italy. ${ }^{4}$ Service Lab, Fleming Research, Milan, Italy. ${ }^{5}$ Department of Nephrology, IRCCS Policlinico San Matteo, Pavia, Italy. 'Service of Laboratory Medicine, IRCCS Policlinico San Donato, Milan, Italy.

Received: 2 October 2013 Accepted: 25 June 2014

Published: 1 July 2014

\section{References}

1. Simmonds HA, Duley JA, Davies PM: Analysis of Purines and Pyrimidines in Blood, Urine and Other Physiological Fluids. In Techniques in Diagnostic
Human Biochemical Genetics: A Laboratory Manual. Edited by Hommes F. NY, USA: Wiley-Liss; 1991.

2. Edvardsson V, Palsson R, Olafsson I, Hjaltadottir G, Laxdal T: Clinical features and genotype of adenine phosphoribosyltransferase deficiency in iceland. Am J Kidney Dis 2001, 38:473-480.

3. Kelley WN, Levy RI, Rosenbloom FM, Henderson JF, Seegmiller JE: Adenine phosphoribosyltransferase deficiency: a previously undescribed genetic defect in man. J Clin Invest 1968, 47:2281-2289.

4. Wyngaarden JB, Dunn JT: 8-Hydroxyadenine as the intermediate in the oxidation of adenine to 2,8-dihydroxyadenine by xanthine oxidase. Arch Biochem Biophys 1957, 70:150-156.

5. Nasr SH, Sethi S, Cornell LD, Milliner DS, Boelkins M, Broviac J, Fidler ME: Crystalline nephropathy due to 2,8-dihydroxyadeninuria: an underrecognized cause of irreversible renal failure. Nephrol Dial Transplant 2010, 25:1909-1915.

6. Simmonds HA, Van Acker KJ, Cameron JS, Snedden W: The identification of 2,8-dihydroxyadenine, a new component of urinary stones. Biochem J 1976, 157:485-487.

7. Tischfield JA, Ruddle FH: Assignment of the gene for adenine phosphoribosyltransferase to human chromosome 16 by mouse-human somatic cell hybridization. Proc Natl Acad Sci U S A 1974, 71:45-49.

8. Broderick TP, Schaff DA, Bertino AM, Dush MK, Tischfield JA, Stambrook PJ: Comparative anatomy of the human APRT gene and enzyme: nucleotide sequence divergence and conservation of a nonrandom CpG dinucleotide arrangement. Proc Natl Acad Sci U S A 1987, 84:3349-3353.

9. Hakoda M, Yamanaka H, Kamatani N, Kamatani N: Diagnosis of heterozygous states for adenine phosphoribosyltransferase deficiency based on detection of in vivo somatic mutants in blood T cells: application to screening of heterozygotes. Am J Hum Genet 1991, 48:552-562.

10. Wilson JM, O'Toole TE, Argos P, Shewach DS, Daddona PE, Kelley WN: Human adenine phosphoribosyltransferase. Complete amino acid sequence of the erythrocyte enzyme. J Biol Chem 1986, 261:13677-13683.

11. Hidaka Y, Tarlé SA, O'Toole TE, Kelley WN, Palella TD: Nucleotide sequence of the human APRT gene. Nucleic Acids Res 1987, 21:9086.

12. Chen J, Sahota A, Laxdal T, Scrine M, Bowman S, Cui C, Stambrook PJ, Tischfield JA: Identification of a single missense mutation in the adenine phosphoribosyltransferase (APRT) gene from five Icelandic patients and a British patient. Am J Hum Genet 1991, 49:1306-1311.

13. Deng $L$, Yang M, Fründ S, Wessel T, De Abreu RA, Tischfield JA, Sahota A: 2,8-Dihydroxyadenine urolithiasis in a patient with considerable residual adenine phosphoribosyltransferase activity in cell extracts but with mutations in both copies of APRT. Mo Genet Metab 2001, 72:260-264.

14. Hidaka Y, Palella TD, OTToole TE, Tarlé SA, Kelley WN: Human adenine phosphoribosyltransferase. Identification of allelic mutations at the nucleotide level as a cause of complete deficiency of the enzyme. J Clin Invest 1987, 80:1409-1415.

15. Sahota A, Chen J, Asaki K, Takeuchi H, Stambrook PJ, Tischfield JA: Identification of a common nonsense mutation in Japanese patients with type I adenine phosphoribosyltransferase deficiency. Nucleic Acids Res 1990, 18:5915-5916.

16. Kamatani N, Terai C, Kuroshima S, Nishioka K, Mikanagi K: Genetic and clinical studies on 19 families with adenine phosphoribosyltransferase deficiencies. Hum Genet 1987, 75:163-168.

17. Hidaka Y, Tarlé SA, Fujimori S, Kamatani N, Kelley WN, Palella TD: Human adenine phosphoribosyltransferase deficiency. Demonstration of a single mutant allele common to the Japanese. J Clin Invest 1988, 81:945-950.

18. Kamatani N, Kuroshima S, Hakoda M, Palella TD, Hidaka Y: Crossovers within a short DNA sequence indicate a long evolutionary history of the APRT*J mutation. Hum Genet 1990, 85:600-604.

19. Kamatani N, Takeuchi F, Nishida Y, Yamanaka H, Nishioka K, Tatara K, Fujimori S, Kaneko K, Akaoka I, Tofuku Y: Severe impairment in adenine metabolism with a partial deficiency of adenine phosphoribosyltransferase. Metabolism 1985, 34:164-168.

20. Bollée G, Dollinger C, Boutaud L, Guillemo D, Bensman A, Harambat J, Deteix P, Daudon M, Knebelmann B, Ceballos-Picot I: Phenotype and genotype characterization of adenine phosphoribosyltransferase deficiency. J Am Soc Nephrol 2010, 21:679-688.

21. Sahota AS, Tischfield JA, Kamatani N, Simmonds HA: Adenine Phosphoribosyltransferase Deficiency and 2,8-Dihydroxyadenine Lithiasis. In The Metabolic and Molecular Bases of Inherited Disease. Edited by Scriver CR, Baudet AL, Sly WS, Valle D. NY, USA: McGraw-Hill; 2001. 
22. Di Pietro V, Peruzza I, Amorini AM, Balducci A, Ceccarelli L, Lazzarino G, Barsotti P, Giardina B, Tavazzi B: Clinical, biochemical and molecular diagnosis of a compound homozygote for the 254 bp deletion- 8 bp insertion of the APRT gene suffering from severe renal failure. Clin Biochem 2007, 40:3-80.

23. Den Dunnen JT, Antonarakis SE: Mutation nomenclature. Curr Protoc Hum Genet 2003, doi:10.1002/0471142905.hg0713s37.

24. Kyte J, Doolittle RF: A simple method for displaying the hydropathic character of a protein. J Mol Biol 1982, 157:105-132.

25. Silva $\mathrm{M}$, Silva $\mathrm{CH}$, lulek J, Thiemann $\mathrm{OH}$ : Three-dimensional structure of human adenine phosphoribosyltransferase and its relation to DHAurolithiasis. Biochemistry 2004, 43:7663-7671.

doi:10.1186/1471-2369-15-102

Cite this article as: Valaperta et al:: Adenine phosphoribosyltransferase (APRT) deficiency: identification of a novel nonsense mutation. BMC Nephrology 2014 15:102.

\section{Submit your next manuscript to BioMed Central and take full advantage of:}

- Convenient online submission

- Thorough peer review

- No space constraints or color figure charges

- Immediate publication on acceptance

- Inclusion in PubMed, CAS, Scopus and Google Scholar

- Research which is freely available for redistribution 